\title{
Fluctuations in Chemical Gelation
}

\author{
Kenji Ohira, Masatoshi Sato and Mahito Kohmoto \\ The Institute for Solid State Physics, the University of Tokyo \\ 5-1-5 Kashiwanoha, Kashiwa, Chiba 277-8581, Japan
}

(Dated: November 13, 2018)

\begin{abstract}
We study a chemical gelation model in two dimensions which includes both monomer aggregations and bond fluctuations. Our numerical simulation shows that a sol-gel transition occurs when an initial monomer concentration is above a critical concentration. Fractal aggregates grow until the sol-gel transition occurs. After the gelation, however, bond fluctuations break the fractal structure and a novel inhomogeneous gel fibre network appears instead. A pore size distribution of the inhomogeneous structure shows the existence of hierarchical structures in the gel phase. It is also found that slow dynamics appear near the critical concentration.

PACS numbers: 82.70.Gg, 05.40.-a, 61.43.Bn, 61.43.Hv
\end{abstract}

\section{INTRODUCTION}

One of the most important characteristics of chemical gels is formation of heterogeneous structure. The structural inhomogeneities affect their physical properties such as permeability, elasticity, and optical properties [1]. Although many studies have been made [2, 3], its dynamical origin has not been elucidated. The difficulty is that they are determined by non-equilibrium dynamics. Since chemical gels are formed by irreversible aggregations between constituent monomers, the structures are frozen in the non-equilibrium gelation processes.

On the other hand, equilibrium properties of gels have been investigated with percolation models [4] which generate equilibrium ensembles. For example, it is wellknown that physical gels formed under annealed conditions are described by equilibrium systems such as correlated percolation models [5, 6, 7]. However, it is not obvious that equilibrium systems can describe the quenched randomness produced by the irreversible aggregations of chemical gels [8].

In this paper, we give a chemical gelation model which includes both monomer irreversible aggregations and bond fluctuations, and numerically study its gelation dynamics in two dimensions. The model introduces bond fluctuations in a similar manner as the bond fluctuation model (BFM) 9] which has been used to model the Rouse dynamics of polymers, and it can simulate polymers and cross-linked gels in an unified framework. By the numerical simulations, a dynamical origin of the

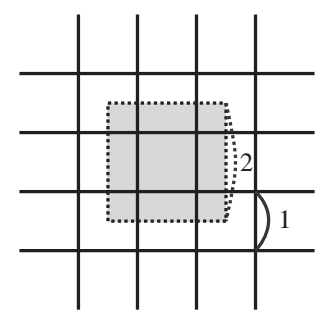

FIG. 1: A monomer on the square lattice. inhomogeneities of chemical gels is reported. We shall show that bond fluctuations drastically change the structures of gels. Although the fractal nature is important in growth kinetics, bond fluctuations break it and lead to a novel inhomogeneous gel fibre network structure.

The paper is organised as follows. Section Idescribes the model and the procedure of numerical simulations. In Sec III we present our numerical results and discuss the gelation dynamics. We show that a critical concentration of gelation exists due to competition between fractal aggregations and bond fluctuations. The inhomogeneous gel networks are investigated in detail and hierarchical structures of the networks are found. We also report that the cluster size distributions below the critical concentration broaden and have a tail with large clusters near the critical concentration. Finally, concluding remarks are given in Sec IV

\section{THE MODEL}

Let us consider $N$ monomers on the $L \times L$ square lattice with the periodic boundary condition. Each monomer is represented by a plaquette shown in Fig 1 and it can jump by one unit lattice. The monomer concentration is

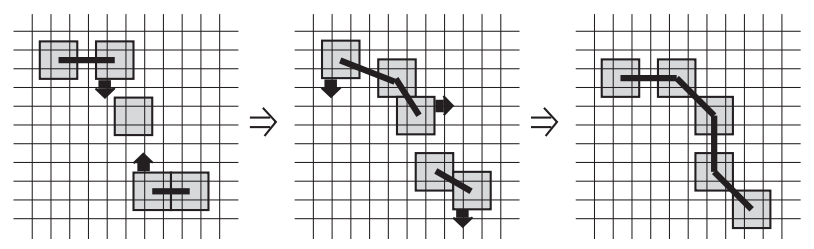

FIG. 2: An aggregation process of two clusters. Gray plaquettes represent monomers and thick lines represent bonds between them. Arrows show randomly chosen directions in which monomers move within $\Delta t=1$. In the final figure, the bond formation condition is met. The two clusters are joined together and become one cluster. 


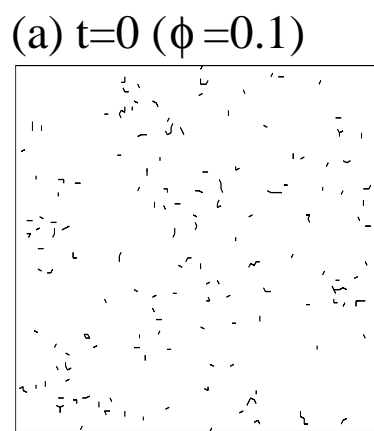

(b) $\mathrm{t}=0(\phi=0.3)$

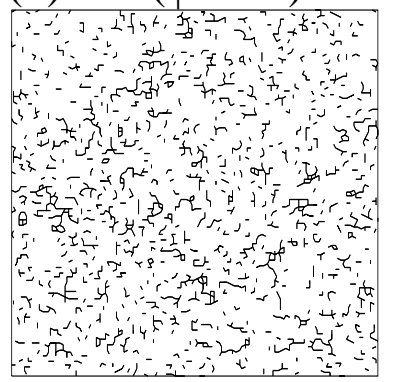

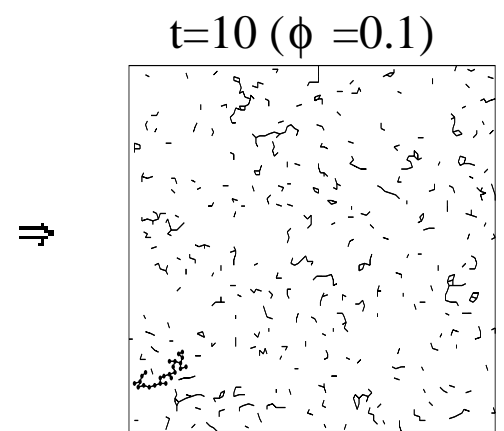
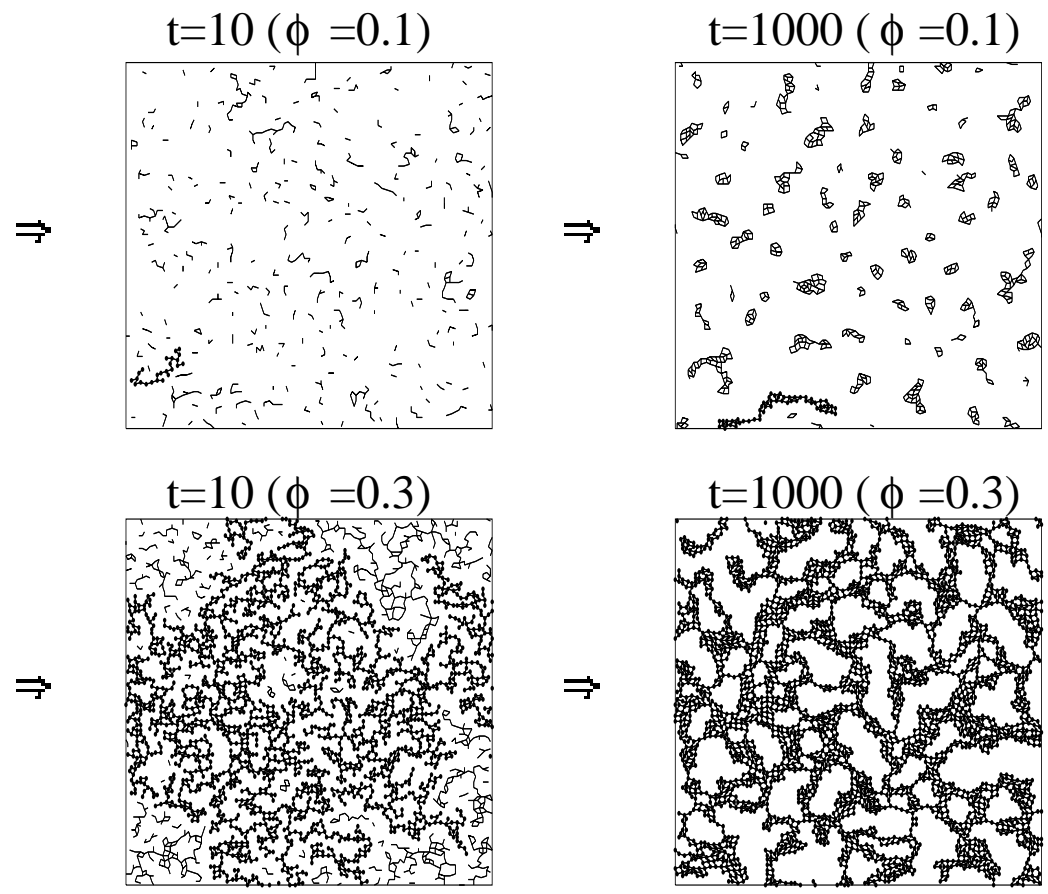

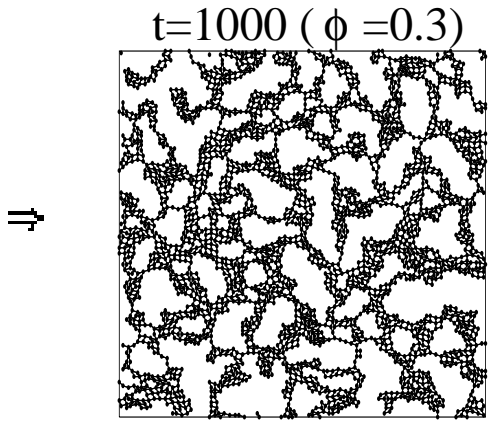

FIG. 3: Snapshots of gelation processes for (a) $\phi=0.1$ and (b) $\phi=0.3$. They are typical examples for high concentrations and low concentrations, respectively. Thin lines represent bonds and black dots represent monomers in the largest cluster. For low concentrations ((a)), clusters have globular structures, and no sol-gel transition occurs even after $t=1000$. For high concentrations $((\mathrm{b}))$, a fractal cluster forms a gel $(t=10)$. After the gelation, however, the intra-cluster bond formation proceeds and the gel becomes inhomogeneous $(t=1000)$.

given by

$$
\phi=\frac{4 N}{L^{2}}
$$

The elementary process of aggregation in our model is formation of a bond between monomers. The bond is created when distance $l_{a}$ between two monomers satisfies

$$
2 \leq l_{a} \leq 3, \quad\left(\text { namely } l_{a}=2, \sqrt{5}, 3\right)
$$

and they both have less than $f$ bonds. ( $f$ is the functionality of the monomer.) The lower bound of $l_{a}$ is determined so as to avoid an overlap between monomers, and the upper bound is the maximal value which guarantees that the bonds never cut through each other during the course of the simulation. As an additional restriction, we forbid formation of a triangle bond where three monomers bond with each other since it leads to an artificial triangle-based ladder structure which prohibits isotropic growth. A bond length $l_{b}$ can change as long as it satisfies

$$
2 \leq l_{b} \leq \sqrt{10},
$$

but a bond itself cannot be broken once it is created. The value $l_{b}$ is determined in a similar manner as $l_{a}$. The variable bond lengths allow movements of clusters. We illustrate an aggregation process of two clusters in Fig 2 ,
We follow the iterative procedure: At $t=0$, we randomly put $N$ monomers on the lattice avoiding overlaps. Then for any pair of monomers we create a bond if the distance between them is one of $l_{a}$ 's in Eq.(2). Next we randomly choose a monomer and move it to a randomly chosen direction by one unit lattice if the monomer does not overlap with others and if the movement is compatible with the bond length restriction (3). If these conditions are not satisfied, we proceed with the next iteration without a movement. After the movement, we create new bonds if the conditions above are met, and proceed with the next iteration. $N$ iterations correspond to a unit physical time $\Delta t=1$. As iterations go, monomers form clusters, and when one of the clusters is bound to itself via the periodic boundary condition, we regard that a gel forms and a sol-gel transition occurs.

The algorithm here is a generalization of BFM so as to include aggregation processes. Indeed, our model reduces to BFM if we take $f=2$, although the bond fluctuation region $l_{b}$ is smaller than that of the original BFM because creation of bonds with length $l_{a}$ bring an additional means of bond crossing. In this paper, we take $f=4$ corresponding to the functionality of a typical crosslinker of cross-linked polymer gels, $N, N$-methylenebis. As well as BFM, each bond in our model effectively represents a group of consecutive C-C bonds along the backbone of the chemically realistic chain. In the same way, each monomer is a coarse-grained monomer which consists of 


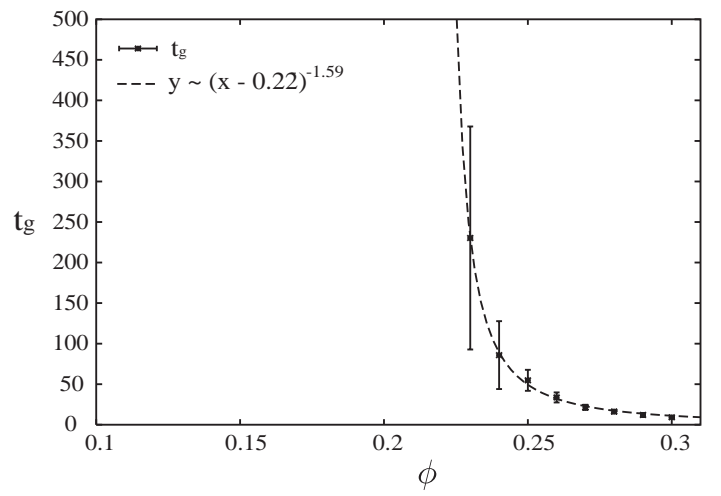

FIG. 4: Gelation time $t_{\mathrm{g}}$ for various concentrations. The dashed line represents the best fitted curve for data. The gelation time shows a divergence at the critical concentration $\phi_{\mathrm{g}} \simeq 0.22$.

a crosslinker and polymers attached to it.

Simulations are performed for concentrations $\phi=0.1-$ 0.3 with the system size $L=200$ unless stated otherwise.

\section{RESULTS}

\section{A. existence of a critical concentration}

Our simulations show a critical concentration $\phi_{\mathrm{g}}$ below which no sol-gel transition occurs. Typical snapshots are shown in Fig 3. In Fig 3(a) the monomer concentration is $\phi=0.1\left(<\phi_{\mathrm{g}}\right)$ and in Fig 3(b) $\phi=0.3\left(>\phi_{\mathrm{g}}\right)$. For $\phi<\phi_{\mathrm{g}}$, the system does not show a sol-gel transition even after very long iterations $(t \leq 10000)$, and we obtain a large number of small globular clusters after all. On the other hand, the system with $\phi>\phi_{\mathrm{g}}$ shows a sol-gel transition (Fig 3(b)). The largest cluster in the system becomes self-connected via the periodic boundary condition and a gel forms in this case. To determine $\phi_{\mathrm{g}}$ we plot the gelation time $t_{\mathrm{g}}$ as a function of $\phi$ in Fig We find that $t_{\mathrm{g}}$ increases as $\phi$ decreases and it becomes infinity at $\phi_{\mathrm{g}} \simeq 0.22$ as

$$
t_{\mathrm{g}} \sim\left(\phi-\phi_{\mathrm{g}}\right)^{-\omega},
$$

where the power index is $\omega \simeq 1.6$. Below $\phi_{\mathrm{g}}$, no solgel transition takes place. The existence of the critical concentration is consistent with experimental results for cross-linked polymer gels.

For $\phi>\phi_{\mathrm{g}}$, the fractal dimension $d_{\mathrm{f}}$ of clusters before gelation is less than the dimension of the system $d$. To show this, we plot in Fig 5 the radii $R$ of the largest clusters in the system at various t's before gelation as a function of their mass $m$. Here $m$ is defined by the number of monomers in the cluster and $R$ is defined by

$$
R^{2}=\frac{1}{2 m^{2}} \sum_{i, j}^{m}\left(\boldsymbol{r}_{i}-\boldsymbol{r}_{j}\right)^{2}
$$

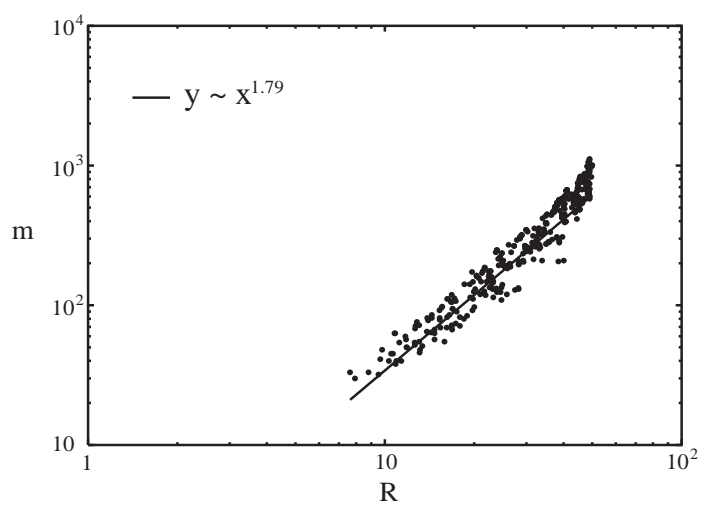

FIG. 5: Radius $R$ of the largest clusters versus their mass $m$ at various times before the sol-gel transition. Here $\phi=0.25$ ( $>$ $\left.\phi_{\mathrm{g}}\right)$. The fractal dimension of the clusters is $d_{\mathrm{f}}=1.79 \pm 0.06$.

where $\boldsymbol{r}_{i}$ is the position vector of the $i$-th monomer in the cluster. For the initial monomer concentration $\phi=$ $0.25\left(>\phi_{\mathrm{g}}\right)$, we find a power law behaviour

$$
m \sim R^{d_{\mathrm{f}}},
$$

where $d_{\mathrm{f}}=1.79 \pm 0.06$. The fractal dimension $d_{\mathrm{f}}$ is almost the same as the corresponding result of the diffusion limited cluster-cluster aggregation (DLCA) model in two dimensions, $d_{\mathrm{f}}^{\mathrm{DLCA}}=1.75 \pm 0.07$ at the same concentration [10, 11, 12]. On the other hand, for $\phi<\phi_{\mathrm{g}}$, the fractal dimension $d_{\mathrm{f}}$ is almost the same as the dimension of the system. In this case, formation of intra-cluster bonds, namely bond formation between monomers in the same cluster, proceeds before gelation and it makes clusters globular as is seen in Fig 3 (a) at $t=1000$.

The difference of the fractal dimensions naturally explain the existence of the critical concentrations $\phi_{\mathrm{g}}$ as follows. When the average mass and radius of clusters are $\langle m\rangle$ and $\langle R\rangle$, respectively, the volume occupied by the clusters $V_{\mathrm{o}}$ is given by

$$
V_{\mathrm{o}} \sim\left(\frac{N}{\langle m\rangle}\right)\langle R\rangle^{d} \sim N\langle R\rangle^{d-d_{\mathrm{f}}}
$$

(Here we have used the relation $\langle m\rangle \sim\langle R\rangle^{d_{\mathrm{f}}}$.) If $V_{\mathrm{o}}$ becomes comparable with the volume of the system

$$
V_{\mathrm{o}} \sim L^{d}
$$

the clusters begin to overlap and a so-gel transition occurs by a process similar to the percolation [13]. For $\phi>\phi_{\mathrm{g}}$, the condition of overlapping (8) is always fulfilled when $\langle R\rangle$ becomes large enough because the inequality $d_{\mathrm{f}}<d$ holds. On the contrary, for $\phi<\phi_{\mathrm{g}}$, the condition (81) is not satisfied. Because of the equation $d \sim d_{\mathrm{f}}, V_{\mathrm{o}}$ rarely depends on $\langle R\rangle$ and never exceeds the volume of the system. Thus no sol-gel transition occurs in this case. 
(a)

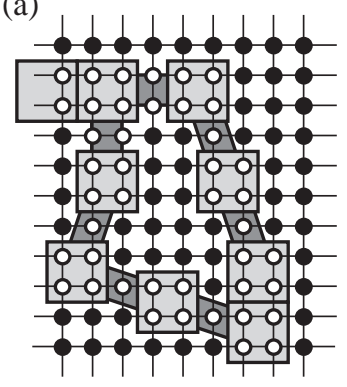

(b)

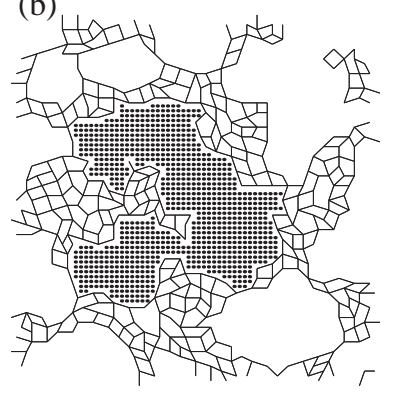

FIG. 6: (a)A schematic representation of a pore. Light and deep gray plaquettes represent monomers and bonds, respectively. Black dots are put on the sites uncovered by the monomers and the bonds. We define a pore as a cluster which consists of the black dots. Figure (b) shows one of the pore. Thin lines represent bonds and the dots are the same as that described above. The size of the pore is defined by the number of the dots

\section{B. an inhomogeneous structure}

The most remarkable feature of our model is the final structure of gels. See Fig[3(b). As shown in Fig 5, gels have a fractal structure similar to the DLCA model until gel formation. Indeed, a snapshot at $t=10$ in Fig[3(b) also shows the fractal structure. However, the fractal structure does not remain after the gel formation. Structure of gels drastically changes at $t=1000$ in Fig 3(b). This is due to formation of intra-cluster bonds after the gel formation. The formation of intra-cluster bonds breaks the fractal structure and makes a novel inhomogeneous gel fibre network structure instead.

In order to investigate the structural changes after the gelation quantitatively, we compare pore size distributions in the gel state at two different times $t=t_{\mathrm{g}}$ and $t \gg t_{\mathrm{g}}$. The pore size is defined as the number of sites in the lattice surrounded by bonding monomers. The precise definition is given as follows. At first, black dots are put on all sites in the lattice, and then the dots covered by monomers are changed to white. Furthermore we define that the bond width is one unit length of the lattice, and the dots covered by the bonds are also changed to white ones. As a result, we obtain clusters of black dots such as Fig 6. We define a cluster of black dots as a pore, and the number of black dots in the cluster as the pore size $s_{\mathrm{p}}$.

Figure 7 shows the pore size distributions for $\phi=$ $0.3\left(>\phi_{\mathrm{g}}\right)$ in two system sizes $L=200$ and 400 at two different times; (a) gelation time $t=10\left(=t_{\mathrm{g}}\right)$ and $(\mathrm{b})$ $t=1000$. We can neglect the part in which the data become flat in large $s_{\mathrm{p}}$ because the flat part depends on system size $L$. At $t=t_{\mathrm{g}}$ the distribution shows two kinds of power law decay with different exponents. For small $s_{\mathrm{p}}$, the distribution behaves as $P\left(s_{\mathrm{p}}\right) \sim s_{\mathrm{p}}^{-\tau}$ with $\tau=1.444 \pm 0.027$, and for large $s_{\mathrm{p}} \tau=1.982 \pm 0.030$. The former value of $\tau$ depends on the monomer concen-
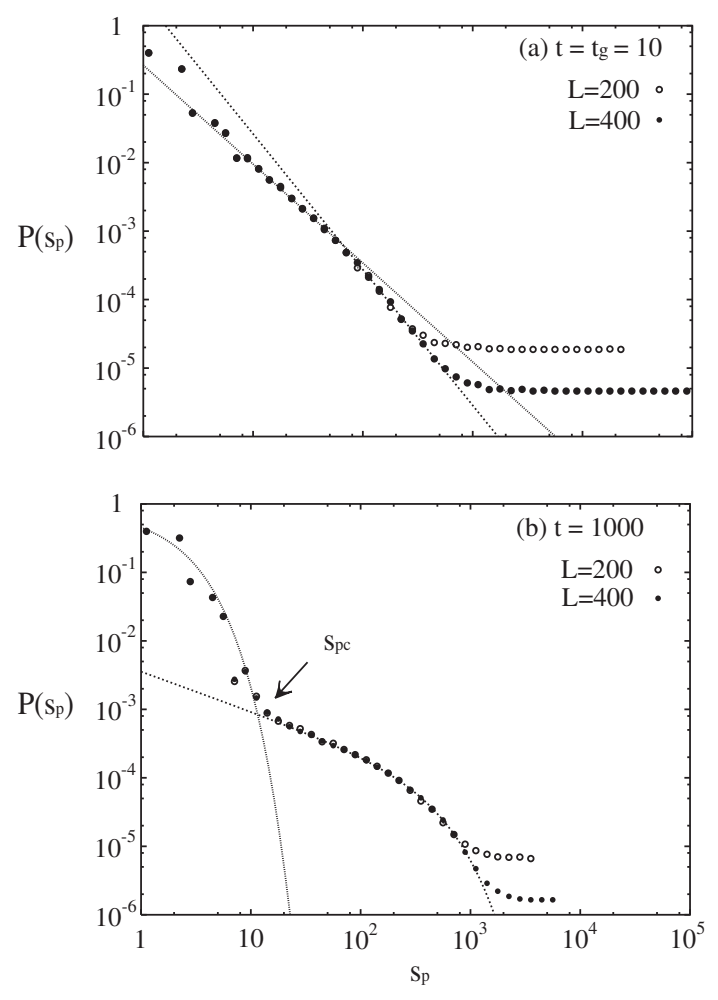

FIG. 7: Pore size distributions $P\left(s_{\mathrm{p}}\right)$ for $\phi=0.3$. (a) At the gelation time, the distribution shows power law decay $P\left(s_{\mathrm{p}}\right) \sim s_{\mathrm{p}}^{-\tau}$ with $\tau=1.444 \pm 0.027$ for small $s_{\mathrm{p}}$ and with $\tau=1.982 \pm 0.030$ for large $s_{\mathrm{p}}$. (b) At $t=1000$, however, the distribution decays exponentially. The crossover size $s_{\mathrm{pc}}$ separates the distribution into monomer-rich and monomerpoor regions.

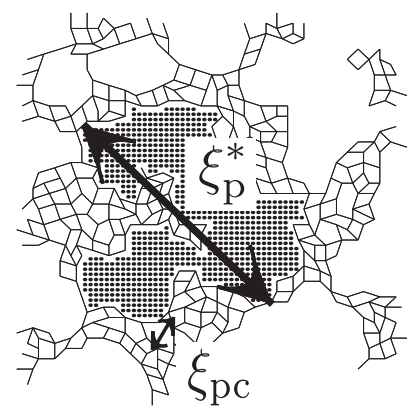

FIG. 8: Two characteristic size scales $\xi_{\mathrm{pc}}$ and $\xi_{\mathrm{p}}^{*}$

tration $\phi$, but the latter $\tau$ does not. Since the latter value is near the Fisher exponent of percolations in two dimensions, $\tau_{\mathrm{F}}=187 / 91 \sim 2.055$ [4], our model belongs to the same universal class in large scales. The distribution is scale invariant and no inhomogeneity is observed. On the other hand, the distribution at $t=1000$ shows a hierarchical structure separated at $s_{\mathrm{p}} \sim s_{\mathrm{pc}}$. See Fig 7(b). For $s_{\mathrm{p}}<s_{\mathrm{pc}}$, the distribution shows an exponential decay, and for $s_{\mathrm{p}}>s_{\mathrm{pc}}$, it can be fitted by the dashed line given by $P\left(s_{\mathrm{p}}\right) \sim s_{\mathrm{p}}^{-\theta} \exp \left(-s_{\mathrm{p}} / s_{\mathrm{p}}^{*}\right)$ with $\theta=0.581 \pm 0.016$ and $s_{\mathrm{p}}^{*}=427.94 \pm 37.08$. The former comes from small pores 


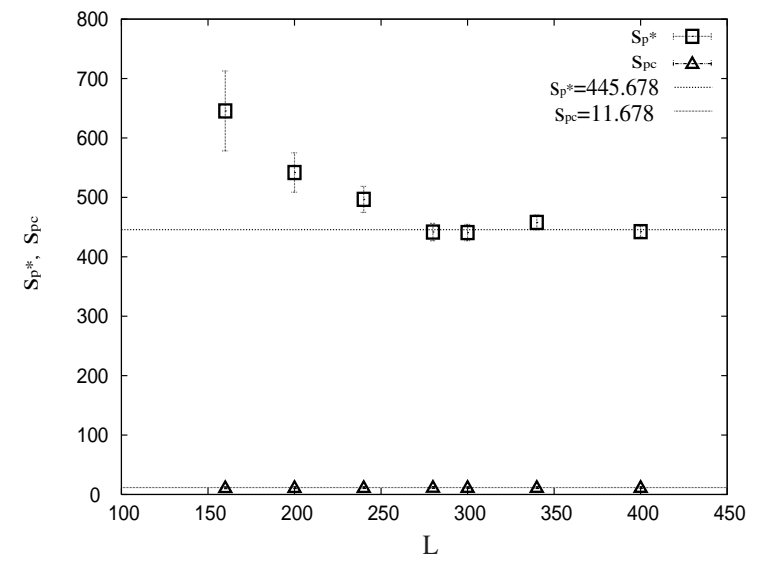

FIG. 9: Size dependence of two characteristic scales $s_{\mathrm{pc}}$ and $s_{\mathrm{p}}^{*}$ of inhomogeneity for $\phi=0.3$ at $t=1000$. They rarely depend on $L$ for large $L$.

in monomer dense regions in Fig (b) at $t=1000$, and the latter from voids in the gel networks. The scale $s_{\mathrm{p}}^{*}$ indicates the characteristic size of the voids. The inhomogeneous structure of the gel networks are characterised by two length scales $\xi_{\mathrm{pc}}$ and $\xi_{\mathrm{p}}^{*}$ corresponding to $s_{\mathrm{pc}}$ and $s_{\mathrm{p}}^{*}$, respectively (Fig 8). In comparison with the distribution at $t=t_{\mathrm{g}}$, the distribution at $t \gg t_{\mathrm{g}}$ contrasts the monomer dense regions with the monomer sparse ones. This phenomena is similar to spinodal decomposition.

We also confirm the existence of $s_{\mathrm{pc}}$ and $s_{\mathrm{p}}^{*}$ in the thermodynamic limit, $L \rightarrow \infty$. In Fig 9, we plot their dependence on the system size $L(\phi=0.3, t=1000)$. It shows that

$$
\begin{array}{ll}
s_{\mathrm{pc}} \simeq 11.678 & \text { for } L \geq 150 \\
s_{\mathrm{p}}^{*} \simeq 445.678 & \text { for } L \geq 280
\end{array}
$$

Therefore, these two scales do not disappear in the thermodynamic limit.

\section{C. cluster size distribution near the critical concentration}

Now we discuss properties near the critical concentration. Figure 10(a) shows the cluster-size distribution near the critical concentration $(\phi=0.2)$ at $t=1000$. For comparison, we also plot the distribution at $\phi=0.1$ in Fig10(b). The data have been taken from 100 samples. We can see that when the concentration $\phi$ is low $(\phi=0.1)$, the distribution is not broad, but near the critical concentration $(\phi=0.2)$, it becomes broader and has a tail toward a large value of $m$. This broadening distribution can be understood as a result of overlapping of clusters near the critical concentration. Namely if the overlapping occurs near the critical concentration, the distribution of clusters are affected by processes similar to percolations which interpolate the overlapping clusters. In general, the percolation processes generally gives a distribution with a tail toward a large value of $m$ [4]. This broadening behaviour can be interpreted as slow dynamics near the gel critical point.

\section{SUMMARY AND DISCUSSIONS}

We examine a chemical gelation model including aggregations of clusters and bond flexibility in two dimensions. The model shows a critical concentration $\phi_{\mathrm{g}}$ below which no sol-gel transition takes place. Above the critical concentration, aggregations before gelation show a fractal structure, but after the sol-gel transition, a novel inhomogeneous gel fibre network structure emerges due to bond fluctuations. From the pore size distributions which characterize the inhomogeneous structure, we find that the structure can be divided into two hierarchical structures; monomer-rich region $\left(r \sim \xi_{\mathrm{pc}}\right)$, and monomer-poor region $\left(r \sim \xi_{\mathrm{p}}^{*}\right)$. This inhomogeneous structure is important to figure out properties of the cross-linked gels. Experimentally, the inhomogeneous structure can be detected as a speckle pattern in the light scattering experiment [14, 15, 16] and a cooperative diffusion of the hierarchical structure can be observed as the so-called gel mode [17]. As is suggested by recent experiments [16], if the speckle pattern is caused by large voids in gel networks, the scale of speckle inhomogeneity is given by $\xi_{\mathrm{p}}^{*}$. At the same time, to observe the speckle pattern in the light scattering experiment, the speckle inhomogeneity should be larger than the laser wavelength $\lambda$ [18], so $\xi_{\mathrm{p}}^{*}$ should be larger than a typical laser wavelength 630 $\mathrm{nm}$. From (9), this gives a lower bound of the size of the coarse-grained monomer, which is estimated to be about $50 \mathrm{~nm}$. In addition, our simulation predicts that no speckle pattern is observed just after the gelation and bond fluctuation is essential to the speckle pattern. We also find that our model realizes the slow dynamics near the critical concentration $\phi \simeq \phi_{\mathrm{g}}$.

Even though our model shows fractal aggregates similar to the DLCA model, its gel is very different from that of the DLCA model. While our model shows no sol-gel transition below the critical concentration, the DLCA model shows a sol-gel transition for any non zero initial concentrations 13. Moreover, our model predicts an inhomogeneous gel network structure, but the DLCA model predicts a fractal structure even after the gelation. Although the gel phase of cross-linked polymers is characterized by the existence of the so-called gel mode [17], the DLCA model can not explain such a specific scale because of its scale invariant nature due to the fractal structure. These differences result from the lack of bond fluctuation in the DLCA model. The DLCA model describes colloidal gels rather than cross-linked polymer gels. For example, the growth by the DLCA in three dimensions is predicted to result in a power-law increase in cluster radius with time, $\langle R\rangle \sim t^{1 / d_{\mathrm{f}}}$, where $\langle R\rangle$ is the average cluster radius and $d_{\mathrm{f}}$ is the fractal dimension. This scaling behaviour is observed excellently by recent exper- 
(a)

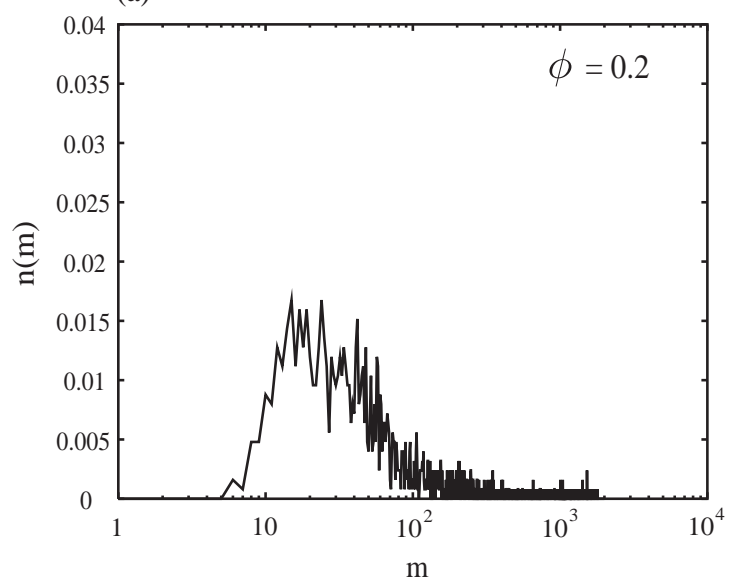

(b)

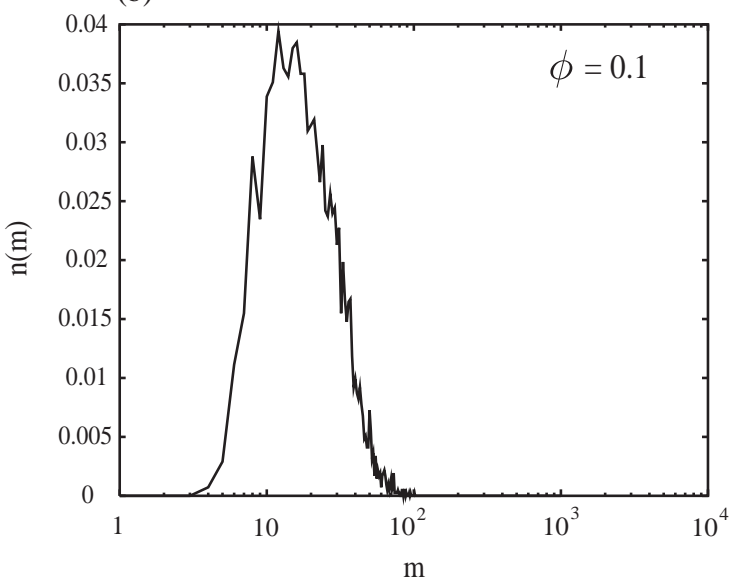

FIG. 10: Cluster size distribution at $t=1000$ below the critical concentration; (a) $\phi=0.2$ and (b) $\phi=0.1$. The vertical axes $n(m)$ denote the number of clusters with mass $m$ over the total number of clusters. The broadening distribution appears at $\phi=0.2$. We take data from 100 samples.

iments of colloidal gels in the International Space Station [19].

In our model the dynamics of monomers connected by bonds are Brownian motions with bond restrictions, and the Brownian force acts on all monomers equivalently regardless of the sizes of clusters they belong to. On the other hand, if one assume that less Brownian force acts on monomers in a larger cluster 20, 21], the inhomogeneous structure cannot be obtained, since intra-cluster bond formation cannot proceed in large clusters due to the low mobility of the constituent monomers. Enough Brownian force acting on monomers in large clusters are needed to form the inhomogeneous structures.

Finally, we would like to point out that the structure of aggregations depends considerably on the functionality of monomers: The system with $f=2$ cannot show a sol-gel transition. Furthermore, in the case of $f=3$, the functionality of monomers becomes easily saturated during gelation processes, thus the intra-cluster bond formation important to the inhomogeneous structure can not proceed enough. Therefore, the minimal functionality showing the inhomogeneous structure is $f=4$, which is the same as that of a typical cross-linker $N, N$-methylenebis.

\section{Acknowledgments}

We thank M. Shibayama and T. Karino for helpful discussions. The computation in this work has been done using the facilities of the Supercomputer Center, Institute for Solid State Physics, University of Tokyo.
[1] Y. Osada, K. Kajiwara, et al., eds., Gels handbook (Academic Press, 2000).

[2] J. Bastide and S. J. Candau, in Physical properties of Polymer Gels, edited by J. P. Cohen Addad (Wiley, New York, 1996), and references therein.

[3] P. Meakin, J. Sol-Gel Sci. Tech. 15, 97 (1999), and references therein.

[4] D. Stauffer and A. Aharony, Introduction to Percolation Theory (Taylor \& Francis, 1994), revised 2nd ed.

[5] P. G. de Gennes, Scaling Concepts in Polymer Physics (Cornell University Press, London, 1985).

[6] A. Coniglio, H. E. Stanley, and W. Klein, Phys. Rev. Lett. 42, 518 (1979).

[7] P. D. Gujrati, J. Chem. Phys. 98, 1613 (1993).

[8] J. E. Martin and J. P. Wilcoxon, Phys. Rev. A 39, 252 (1989).

[9] I. Carmesin and K. Kremer, Macromol. 21, 2819 (1988).

[10] P. Meakin, Phys. Rev. Lett. 51, 1119 (1983).

[11] M. Kolb, R. Botet, and R. Jullien, Phys. Rev. Lett. 51, 1123 (1983).
[12] M. Kolb and H. J. Herrmann, J. Phys. A: Math. Gen. 18, L435 (1985).

[13] J. C. Gimel, D. Durand, and T. Nicolai, Phys. Rev. B 51, 11348 (1995).

[14] E. S. Matsuo, M. Orkisz, S. Sun, Y. Li, and T. Tanaka, Macromol. 27, 6791 (1994).

[15] M. Shibayama, Macromol. Chem. Phys. 199, 1 (1998).

[16] Y. Zhao, G. Zhang, and C. Wu, Macromol. 34, 7804 (2001).

[17] T. Tanaka, L. O. Hocker, and G. B. Benedek, J. Chem. Phys. 59, 5151 (1973).

[18] J. C. Dainty, ed., Laser Speckle and Related Phenomena (Springer-Verlag Berlin Heidelberg New York, 1975).

[19] S. Manley et al., Phys. Rev. Lett. 93, 108302 (2004).

[20] R. Jullien and A. Hasmy, Phys. Rev. Lett. 74, 4003 (1995).

[21] A. Hasmy, E. Anglaret, R. Thouy, and R. Jullien, J. Phys. I France 7, 521 (1997). 